\title{
CONDICIONES, REGULACIONES Y EXPERIENCIAS DE TRABAJO DOCENTE: EL CASO DE LA UNIVERSIDAD NACIONAL DEL SUR
}

\author{
Julieta Rodera* \\ Universidad Nacional del Sur, Argentina \\ juli.rodera@gmail.com \\ Verónica Soledad Walker ** \\ Universidad Nacional del Sur, Argentina \\ veronica.walker@uns.edu.ar
}

Recibido: 10/08/2020 Aceptado: 15/10/2020

\section{Resumen}

El artículo presenta avances de una investigación en curso sobre las condiciones, regulaciones y experiencias de trabajo docente en la Universidad Nacional del Sur (UNS, Argentina). Se comparte parte del análisis de 21 entrevistas semi-estructuradas realizadas a docentes de distintas categorías y dedicación de cuatro unidades académicas y se recuperan datos proporcionados por una encuesta estructurada aplicada a una muestra de 516 docentes de la institución.

El trabajo se divide en cuatro apartados. En el primero se presentan las perspectivas teóricas asumidas como puntos de partida para el estudio. En el segundo se realiza una breve descripción de la UNS, institución donde se realizó el estudio. En el tercero se aborda el análisis de las entrevistas y las encuestas realizadas, bajo los siguientes ejes: iniciarse como docente universitario, trabajar en la universidad, virtualización forzada del Trabajo Docente Universitario en el contexto de pandemia por COVID-19. Por último, se esboza una serie de reflexiones finales.

Palabras clave: Trabajo Docente Universitario - Experiencias - Condiciones laborales - Virtualidad.

\footnotetext{
* Estudiante de la Licenciatura en Ciencias de la Educación de la Universidad Nacional del Sur (UNS, Argentina). Actualmente se encuentra realizando la tesina de grado y con una Beca para Alumnos Avanzados de la Universidad Nacional del Sur. Ha participado en Proyectos de Grupos de Investigación dentro del Departamento y actualmente integra un proyecto sobre formación y trabajo docente en la universidad. Ha presentado ponencias en congresos y jornadas académicas.

** Doctora en Didáctica y Organización Educativa por la Universidad de Málaga (UMA, España), Especialista en Ciencias Sociales con Mención en Evaluación e Investigación Educativa por la Universidad Nacional de Quilmes (UNQ, Argentina), Licenciada y Profesora en Ciencias de la Educación por la Universidad Nacional del Centro de la Provincia de Buenos Aires (UNCPBA, Argentina). Actualmente se desempeña como Profesora Adjunta por concurso de los Departamentos de Humanidades y Economía para la Licenciatura en Ciencias de la Educación de la Universidad Nacional del Sur (UNS, Argentina). Ha participado en diversos proyectos de investigación y actualmente dirige un Proyecto Grupal de Investigación radicado en la UNS sobre formación y trabajo docente en la universidad. Es autora y coautora de capítulos de libros, artículos de revistas con referato y ponencias en eventos científicos. Sus líneas de investigación son las políticas universitarias de evaluación, la formación universitaria y el trabajo docente en la universidad.
} 


\title{
CONDITIONS, REGULATIONS AND TEACHING WORK EXPERIENCES: THE CASE OF THE UNIVERSIDAD NACIONAL DEL SUR
}

\begin{abstract}
The article presents advances of an ongoing investigation on the conditions, regulations and experiences of teaching work at the Universidad Nacional del Sur (UNS, Argentina).

Part of the analysis of 21 semi-structured interviews carried out with teachers of different categories and dedication of four academic units is shared and data provided by a structured survey applied to a sample of 516 teachers of the institution is recovered.

The work is divided into four sections. The first presents the theoretical perspectives assumed as starting points for the study. The second contains a brief description of the UNS, the institution where the study was carried out. The third deals with the analysis of the interviews and surveys conducted, as follows: starting as a university teacher, working at the university, forced virtualization of University Teaching Work in the context of a COVID-19 pandemic. Finally, a series of final reflections is outlined.
\end{abstract}

Keywords: University Teaching Work - Experiences - Working conditions - Virtuality.

\section{Introducción}

El trabajo docente universitario como objeto de estudio comenzó a cobrar relevancia en Argentina hacia finales del siglo XX y principios del XXI (Chiroleu, 2002; Tello et. al., 2004; García de Fanelli, 2008; Fernández Lamarra y Pérez Centeno, 2011). Es un campo de estudio considerado hasta hace poco de vacancia y que actualmente se encuentra en desarrollo (Pérez Centeno, 2017; Walker, 2019). Asimismo, es un tema que está presente en la agenda nacional por los cambios que introdujo en 2015 la aprobación del Convenio Colectivo de Trabajo (CCT) para el sector universitario en nuestro país y actualmente por las transformaciones introducidas por la virtualización forzada de las actividades académicas o la enseñanza remota de emergencia en los contextos de aislamiento social y preventivo obligatorio (ASPO) y distanciamiento social preventivo y obligatorio (DISPO) por la pandemia de COVID-19.

El presente artículo expone parte de los resultados de un trabajo de investigación en curso sobre las condiciones, regulaciones y experiencias de trabajo docente universitario en un escenario institucional particular: la Universidad Nacional del Sur (UNS, Argentina) ${ }^{1}$. Se trata de un estudio de caso único de carácter intrínseco (Stake, 1999) que integra análisis estadístico y documental, entrevistas exploratorias semi-estructuradas a docentes de cuatro unidades académicas y los resultados de una encuesta estructurada con preguntas cerradas y abiertas aplicada a una muestra representativa del plantel docente de la universidad. Para la triangulación y análisis de estos instrumentos se considera una serie de criterios que dan cuenta de las posiciones en el espacio universitario de las/os docentes entrevistadas/os y encuestadas/os: área disciplinar de pertenencia, categoría y dedicación docente, género autopercibido y antigüedad en la docencia universitaria. Se focalizará en el análisis de 21 entrevistas y se recupera parte de los datos proporcionados por la encuesta.

El artículo se organiza en cuatro apartados. En el primero, se desarrollan las perspectivas teóricas asumidas como puntos de partida para el estudio. En el segundo, se presenta una breve descripción de la Universidad Nacional del Sur, institución donde se lleva a cabo el estudio. En el tercero, se plantea el análisis de las entrevistas a docentes de distintas áreas disciplinares, focalizando en tres dimensiones: los inicios de la carrera docente, la experiencia de trabajo en la UNS y los cambios provocados por la virtualización en el ejercicio cotidiano de la tarea docente. Finalmente, se presentan algunas reflexiones finales.

\section{Perspectivas teóricas sobre el trabajo docente universitario}

La tarea docente en la universidad ha sido objeto de diversas conceptualizaciones que obedecen a las perspectivas teóricas asumidas. En esta investigación se recupera la categoría de trabajo y se concibe la institución universitaria como un espacio laboral atravesado por regulaciones de distintos actores y a distintos niveles del campo universitario. Se entiende que en este entramado de condiciones y regulaciones las/os docentes construyen particulares experiencias y sentidos sobre su trabajo en la universidad.

Se concibe la universidad como un campo social, como "espacios de juego históricamente constituidos con sus instituciones específicas y sus leyes de funcionamiento propias" (Bourdieu, 1988,

${ }^{1}$ Se trata del trabajo realizado como parte de una tesina de grado para obtener el título de Licenciatura en Ciencias de la Educación en el marco del programa de Becas para Alumnos Avanzados de la Universidad Nacional del Sur (UNS) y del Proyecto Grupal de Investigación (PGI) "Universidad, formación y trabajo docente: condiciones, regulaciones y prácticas", SGCyT-UNS. Código: 24/ZI58. 
REVISTA DE LA ESCUELA DE CIENCIAS DE LA EdUCACIÓN, AÑO 17, NRO. 16, VOL. 2, JULIO A DICIEMBRE DE 2021. PÁGINAS 171-180. ISSN 2362-3349 (EN LÍNEA).CONDICIONES, REGULACIONES Y EXPERIENCIAS DE TRABAJO DOCENTE: EL CASO DE LA UNIVERSIDAD NACIONAL DEL SUR. JULIETA RODERA. VERÓNICA SOLEDAD WALKER.

p.108). En este sentido, la universidad puede entenderse como un campo de lucha que tiene reglas propias, donde se contraponen intereses y hay varios capitales en juego. Esto se vislumbra en la dinámica institucional, donde coexiste diversidad de actores y lógicas. Para Bourdieu, la universidad es "el espacio de una disputa para determinar las condiciones y los criterios de la membresía legítima y la jerarquía legítima, de determinar qué propiedades son pertinentes, efectivas para funcionar como capital capaz de garantizar los beneficios específicos del campo" (Krotsch, 2001, p. 65). En el campo universitario argentino hay organismos como la Comisión Nacional de Evaluación y Acreditación Universitaria (CONEAU), el Consejo Interuniversitario Nacional (CIN) y la Secretaría de Políticas Universitarias (SPU), además de los organismos de ciencia y tecnología como el Consejo Nacional de Investigaciones Científicas y Técnicas (CONICET), que definen políticas y programas que orientan las prácticas de los actores en el campo universitario. Esta orientación puede analizarse desde la categoría de regulación que, siguiendo a Barroso (2005), constituye un proceso constitutivo de todo campo cuya función principal es garantizar el equilibrio y la coherencia pero también permitir la transformación. Es decir, se trata de un proceso que representa las reglas que guían el funcionamiento de un sistema pero también también los necesarios reajustes que entran en juego por la diversidad de los actores involucrados, sus posiciones, intereses y estrategias. Para el autor, la interacción de múltiples dispositivos reguladores permite hablar de 'multirregulación'. Además, el concepto de regulación permite describir dos tipos de fenómenos diferenciados pero interdependientes: los modos como son producidas y aplicadas las reglas que orientan la acción de los actores y los modos como esos mismos actores se apropian de ellas y las transforman.

Los docentes universitarios, comenzaron a ser considerados actores clave en el análisis de los sistemas de educación superior a partir de la década de 1980. A nivel internacional, encontramos estudios pioneros sobre el profesorado universitario y sus espacios de trabajo que, desde la categoría de profesión, colocan a los académicos como objeto de investigación (Clark, 1983; Becher, 1989; Perkin, 1984; Boyer et ál., 1994). En América Latina, los estudios de Bruner (1985), Bruner y Flisfisch (1989) señalan las modificaciones en las condiciones del mundo del trabajo académico y la constitución de un mercado ocupacional académico a partir del proceso de masificación de las universidades iniciado en la década de 1960. En Argentina, la diversidad identitaria del profesorado universitario ligada a las particulares adscripciones disciplinares, configuró hacia la década de 1990 dos líneas de investigación sobre docencia universitaria: una centrada en las prácticas de enseñanza y otra en los efectos de las políticas de evaluación en el trabajo académico. Ambas vías de entrada, contribuyeron al reconocimiento y constitución de la docencia universitaria como un objeto de estudio propio que exige categorías de análisis y formas de abordaje específicas (Walker, 2019). A partir de la primera década del 2000, se asiste a la emergencia de estudios sobre la profesión académica que significaron un avance importante en la caracterización de esta categoría ocupacional (García de Fanelli, 2008; Fernández Lamarra y Pérez Centeno, 2011; Marquina y Fernández Lamarra, 2008; Marquina, 2013).

Sin embargo, aunque en los últimos años comienzan a aparecer investigaciones referidas a las condiciones de trabajo y los cambios en la cultura académica, "el trabajo como tal ha sido y aún es, escaso tema de análisis y reflexión en la vida universitaria” (Martínez, 2013, p. 48).

En la presente investigación, asumimos la docencia universitaria desde la categoría de trabajo, como un trabajo de tipo intelectual. El trabajo docente universitario es una práctica social, históricamente situada y relacional que se despliega en una trama compleja de condiciones, regulaciones y tensiones y que coloca a las/os docentes en una red de múltiples dependencias recíprocas, en una red de relaciones con otras/os, con diferentes saberes, con instituciones particulares y con la sociedad en general. Consideramos importante recuperar esta categoría en un espacio organizacional que en general no suele ser entendido como un espacio laboral y en el que los docentes no suelen percibirse como trabajadores.

Martínez destaca ciertas particularidades del trabajo en la universidad. En primer lugar enfatiza que "las rutinas y regularidades en el trabajo en la universidad tienen ritmos desiguales, fracturas y escisiones. Lo real del trabajo es lo que se realiza más allá de lo prescripto o a pesar de él" (2013, p. 50), y por lo cual, concibe como necesario visibilizar lo no-remunerado dentro del trabajo asalariado, incluso en la docencia cuando se ocupan -con o sin alumnos- más horas que las reglamentarias sin reclamo de pago. Asimismo resalta que "hay tiempos extra, voluntarios, individuales y grupales, en horas y días laborables o feriados: informes de investigación, revisiones de tesis de grado o trabajos para publicar, consultas preexamen, no siempre obligatorios (tanto para estudiantes como docentes)" (2013, p. 57). De igual manera, refiere a otros tipos de trabajos que resultan "invisibles" en la universidad como trabajos voluntarios políticos, sociales o culturales.

Por lo expuesto, cobra sentido indagar las experiencias que atraviesan diariamente las/os docentes en la universidad. Entendiendo que las regulaciones y lo prescripto operan a través de las vivencias singulares que no se reducen a los acontecimientos, sucesos y/o rutinas sino a lo que éstos significan e importan para los sujetos, es decir, cómo son re-significados por cada docente. Para Larrosa (2003) la experiencia como "lo que me pasa", es una manera de habitar el mundo y de vivirlo, que está cargado de sentidos. La experiencia, como dice el autor no podría generalizarse pero tampoco podría pensarse que existen experiencias enteramente individuales, con esto se hace referencia a que toda experiencia, toda vivencia está enmarcada en un contexto, en una estructura. Es decir, eso que le pasa al sujeto forma parte de una realidad en disputa que lo interpela (Salguero Myers, 2016). 
REVISTA De LA Escuela de CiENCIAS DE LA EduCACIÓN, AÑO 17, NRO. 16, VOL. 2, JULIO A DiCIEMBRE DE 2021. PÁGINAS 171-180. ISSN 2362-3349 (EN LÍNEA). CONDICIONES, REGULACIONES Y EXPERIENCIAS DE TRABAJO DOCENTE: EL CASO DE LA UNIVERSIDAD NACIONAL DEL SUR. JULIETA RODERA. VERÓNICA SOLEDAD WALKER.

Es en este sentido que resulta indispensable pensar a las/os docentes como sujetos de experiencia, que son "construidos en y a través de las mismas, de las vivencias que les llenan de contenido y de los sentidos que las organizan" (Guzmán Gómez y Saucedo Ramos, 2015, p. 1032). Las experiencias de trabajo docente en la universidad ocurren siempre ligadas a un contexto institucional y social particular, donde entran en juego las reglas y luchas propias del campo y de sus relaciones con otros campos.

\section{Breve caracterización del caso estudiado: la Universidad Nacional del Sur}

La Universidad Nacional del Sur (UNS) se fundó el 5 de enero de 1956 en la ciudad de Bahía Blanca, provincia de Buenos Aires. Tuvo su origen en el "Instituto Tecnológico del Sur", creado en octubre de 1946 y dependiente de la Universidad Nacional de La Plata. El mismo fue instaurado ante la necesidad de brindar estudios del nivel superior para aquellos estudiantes que querían incorporarse como profesionales a la naciente industria de la zona del sudoeste bonaerense.

Esta casa de estudios organizó su estructura académica por Departamentos, organización que tiene origen en las universidades norteamericanas, y que se diferencia del modelo clásico de cátedras y facultades, propio de las universidades europeas.

El sistema departamental "se caracteriza por disponer del monopolio sobre las tareas de docencia, investigación y extensión de un campo particular del conocimiento" (Toribio, 1999, p.18). En este modelo se destaca la flexibilidad en el uso del personal académico, el conocimiento dividido en diferentes áreas (por departamentos), la descentralización del poder en la institución y la posibilidad de unificar estudiantes de diferentes carreras en un mismo curso (Follari y Soms, 1980). Tiene una estructura administrativa menor que permite flexibilidad y transversalidad en el conocimiento. De ahí que, las unidades académicas no deben crear para sus carreras las materias que otras dictan para las suyas, lo que permite a las/os estudiantes cursarlas en ellos; "brindando de esta manera una mayor cohesión a la estructura universitaria y tendiendo a lograr economía de esfuerzos y de medios materiales" (Estatuto UNS, 1996, Art. 5).

Actualmente la UNS cuenta con dieciséis Departamentos ${ }^{4}$ de diferentes áreas del conocimiento. La oferta académica que brinda tiene un notorio carácter científico-tecnológico, propio de su origen, ya que predominan las disciplinas de Ciencias Exactas y Naturales por sobre las de Humanidades y Ciencias Sociales.

En referencia a la cantidad de estudiantes que concurren a dicha universidad, según datos de los Anuarios Institucionales, son 23.901 las/os estudiantes de carreras de grado, 2.696 de pregrado y 1.724 de posgrado. La mayoría de las/os estudiantes de grado provienen de la región, siendo un $84 \%$ de la provincia de Buenos Aires, un 9,4\% de Río Negro y un 3\% de La Pampa.

Con respecto al plantel docente, según datos del Anuario UNS 2019, la institución cuenta con un total de 2.922 cargos docentes. Tal como se muestra en el Cuadro $\mathrm{N}^{\circ} 1$, la mayor proporción de cargos docentes corresponde a dedicación simple (67\%), concentrándose este tipo de dedicación en la categoría más baja del escalafón correspondiente a Ayudantes de Docencia A, reproduciendo la estructura piramidal que se observa en la mayoría de las universidades argentinas. Las dedicaciones exclusivas representan el $21,5 \%$ superando la media nacional. Al respecto, según datos de los Anuarios Estadísticos de la Secretaría de Políticas Universitarias (SPU) en el período 1998-2015 la media histórica de cargos con dedicación exclusiva en el conjunto de universidades nacionales (UUNN) es de 13\%, concentrándose en los cargos de profesores.

\begin{tabular}{|c|c|c|c|c|c|}
\hline & Exclusiva & $\begin{array}{l}\text { Semi } \\
\text { Exclusiva }\end{array}$ & Simple & Total & \\
\hline Profesor Titular & 82 & 6 & 19 & 107 & 53 \\
\hline Profesor Asociado & & 10 & & 150 & \\
\hline
\end{tabular}

\footnotetext{
4 Agronomía; Geografía y Turismo; Biología, Bioquímica y Farmacia; Geología; Ciencias de la Administración; Humanidades; Ciencias de la Salud; Ingeniería; Ciencias e Ingeniería de Computación; Ingeniería Eléctrica y de Computadoras; Derecho; Ingeniería Química; Economía; Matemática; Física; Química.
} 


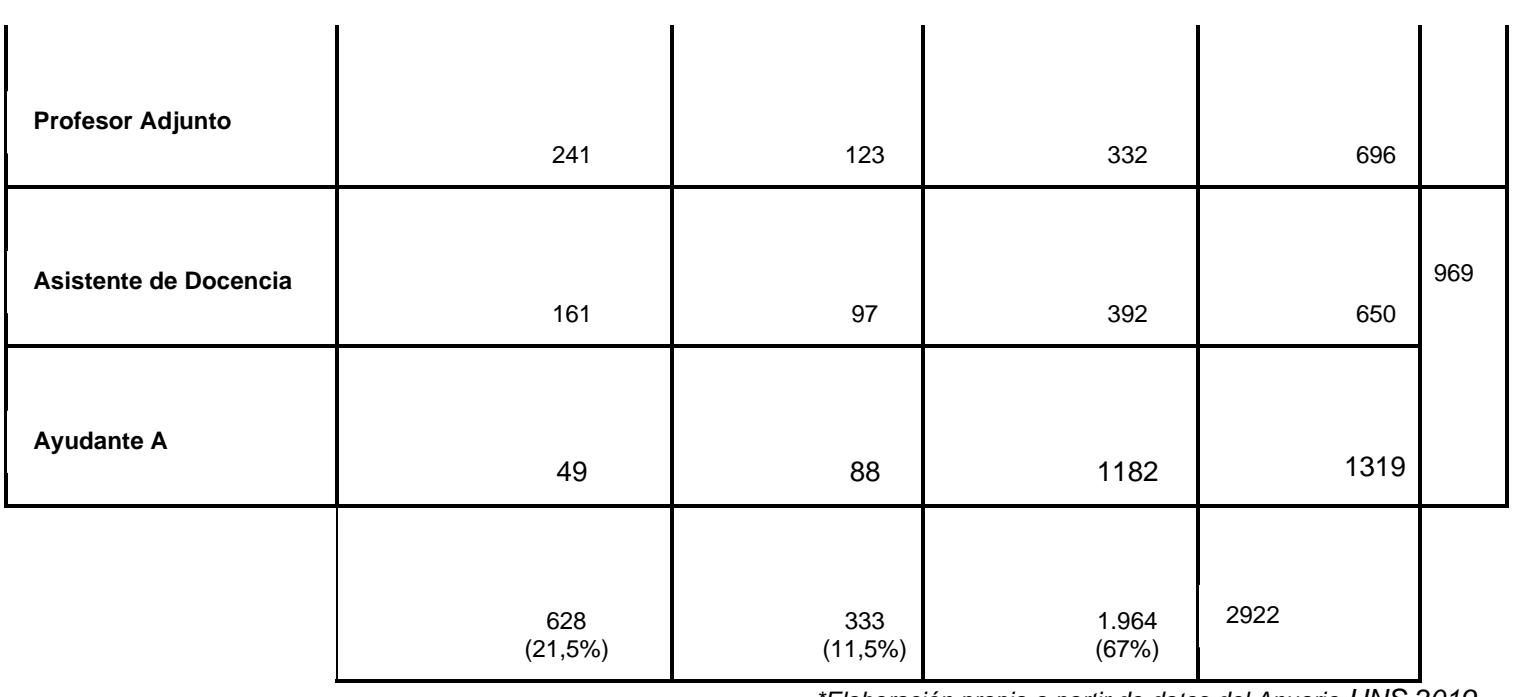

*Elaboración propia a partir de datos del Anuario UNS 2019.

Si se focaliza en las dedicaciones docentes según unidad académica, se observa una gran heterogeneidad como es el caso del Departamento de Derecho que cuenta con un $96 \%$ de cargos de dedicación simple (96\%), al igual que Ciencias de la Administración (85\%) y Ciencias de la Salud (85\%) que también siguen dicha tendencia. Estos son Departamentos que se caracterizan por su perfil profesionalista, los cuales, a su vez, según planean Walker et ál. (2016), son los que presentan menor proporción de docentes categorizados en el Programa de Incentivos: Derecho (14,86\%), Ciencias de la Administración $(25,73 \%)$ y Ciencias de la Salud $(15,25 \%)$.

Siguiendo con lo planteado por Walker et ál. (2016), del total de docentes de la UNS un 48,43\% tiene título de posgrado, de los cuales la mayoría corresponde a Doctorado. Distinguiendo por Departamentos, los que tienen mayor proporción de docentes con título de Doctorado corresponden a Biología, Química y Farmacia (63,01\%), Ingeniería Química (61,39\%), Física (61,04\%) y Química (60,61\%). Estas cifras sobresalen del escenario nacional donde solo representan un $20 \%$ y se acerca al promedio mundial, donde el título de posgrado es considerado como requisito (Marquina, 2013). Cabe aclarar que en el caso de la UNS, los Departamentos con mayor proporción de docentes que no cuentan con título de posgrado son quienes presentan el menor porcentaje de docentes categorizados, como se mencionó anteriormente.

Las diferencias que derivan de los distintos perfiles y culturas organizacionales son puestas de manifiestos también en los relatos de las/os docentes acerca de sus experiencias de trabajo en la universidad.

En el año 2020, en el marco de las medidas de emergencia sanitaria establecidas el 20 marzo de 2020 por el gobierno nacional, la UNS decidió postergar el inicio del primer cuatrimestre inicialmente definido para el 25 de marzo. Así, las actividades de enseñanza de grado iniciaron el 13 de abril de manera virtual. Esto llevó a que la mayoría de los docentes experimenten una "virtualización forzada" de su trabajo docente, modificándose drásticamente sus condiciones de trabajo.

\section{Experiencias de trabajo en la universidad desde la perspectiva de las/os docentes}

En este apartado se presenta el análisis de 21 entrevistas semi-estructuradas realizadas a docentes de distintas unidades académicas de la UNS -Agronomía, Economía, Ciencias de la Salud y Humanidades- seleccionadas según el área disciplinar y el perfil de egresado al que aspiran. A su vez, para la selección de las/os entrevistadas/os se tuvieron en cuenta las categorías y dedicaciones de los cargos que ocupan y el momento de la trayectoria académica en el que se encuentran.

Las entrevistas tuvieron lugar entre los meses de mayo y julio de 2020 y algunos de los ejes de indagación fueron: el ingreso al sistema universitario, la actividad de docencia y su trabajo en la universidad, las regulaciones que atraviesan sus prácticas docentes, las experiencias y sentidos que construyen sobre su trabajo y los cambios provocados por la virtualización en tiempos de pandemia.

En algunos ejes, el análisis de las entrevistas se triangula con los datos obtenidos de una encuesta estructurada aplicada a una muestra de 516 docentes de la institución. De esta manera, se buscará reconocer aspectos comunes y diversos de las experiencias de trabajo según las heterogéneas posiciones desde las que cada docente ejerce su tarea.

\section{Iniciarse como docente universitario}

En el transcurrir de las entrevistas, una de las cuestiones que se mencionó de forma recurrente es el ingreso o el acceso al sistema universitario y a la UNS en particular, es decir cómo habían sido los inicios como docentes en la institución. Las/os entrevistadas/os manifestaron diversas formas de acceso a los 
REVISTA DE LA ESCUELA DE CIENCIAS DE LA EdUCACIÓN, AÑO 17, NRO. 16, VOL. 2, JULIO A DICIEMBRE DE 2021. PÁGINAS 171-180. ISSN 2362-3349 (EN LÍNEA). CONDICIONES, REGULACIONES Y EXPERIENCIAS DE TRABAJO DOCENTE: EL CASO DE LA UNIVERSIDAD NACIONAL DEL SUR. JULIETA RODERA. VERÓNICA SOLEDAD WALKER.

cargos, ya sea ordinario, interino o por contrato, las diferentes representaciones que tenían acerca de la universidad y el trabajo en ésta, los miedos e incertidumbres, los recorridos que tuvieron que transitar para poder presentarse a un concurso como también los motivos por los que decidieron ingresar a la universidad.

Respecto a las formas de ingreso, las/os entrevistadas/os relataron singulares vivencias en sus inicios como docentes en la UNS. Algunas/os accedieron a cargos docentes de carácter ordinario a través de concurso abierto, de antecedentes y oposición. Otras/os lo hicieron a través de llamados a inscripción para cargos interinos. Muchas/os señalaron sus inicios en la UNS a través de contratos temporarios, pero que terminaron renovándose por muchos años. Algunas/os recordaron sus experiencias como Ayudantes $\mathrm{B}$ (cargo docente rentado para estudiantes que tienen un año de duración), incluso por años. Respecto a los cargos docentes ad honorem, en el caso de la UNS, sólo están contemplados para las designaciones de profesores extraordinarios que constituyen una distinción honorífica, a diferencia de otras universidades nacionales en las que persiste este tipo de contratación, siendo incluso el mecanismo mayormente implementado para el acceso a la carrera docente (Rikap, 2016).

Así como se advierte una diversidad en las formas de ingreso como docente, también se reconoce una importante diversificación en las trayectorias académicas. Es decir, más allá que el escalafón de categorías docentes estipula un recorrido gradual por cargos con grados crecientes de responsabilidad, las trayectorias docentes lejos están de ser lineales. Hay docentes que han podido ascender de manera escalonada, de categoría en categoría -desde Ayudante B a Profesor Titular-, mientras muchas/os otras/os se encuentran en un mismo cargo por años sin posibilidades de promoción. Esta realidad, que constituye uno de los principales obstáculos de la carrera académica en la mayoría de las universidades de nuestro país (García de Fanelli y Moguillansky, 2014) es adjudicada a la falta de presupuesto o razones administrativas por las que no se abren los llamados a concursos o por 'no ajustarse' a ciertos requerimientos. Como expresa este docente: "en la regla no escrita la investigación sigue siendo la niña mimada y es justamente donde yo tengo limitación, entonces no es casual que todavía a ocho años de jubilarme soy Profesor Adjunto" (Docente Dpto. de Agronomía, 22 años de antigüedad). Situación que genera -en muchos casos- una sobrecualificación, ya que cada vez hay más docentes que cuentan con títulos de posgrado, con carrera de investigación en organismos como CONICET, y sin embargo, siguen sin posibilidades de ascenso en la carrera docente universitaria, permaneciendo en la misma categoría por años, tal como manifestaron varias/os de las/os entrevistadas/os.

Otro factor condicionante en el ingreso a la universidad, mencionado por las/os docentes, es el Departamento donde se radica el cargo debido a las particularidades que devienen del perfil de egresado al que aspiran, las lógicas propias de cada campo disciplinar y las dinámicas que se fueron sedimentando en este nivel de la organización.

Por último, una consideración que también cabe mencionar es el motivo por el cual ingresaron a la universidad. En algunos casos se dio por ser el espacio o la salida laboral más cercana, incluso como alternativa al sector privado. En otros fue una decisión impensada, es decir, su carrera de base no era la docencia, sin embargo encontraron un lugar en ésta y se desarrollaron en su disciplina. Otras/os la eligieron por el disfrute de la docencia en sí, no por el nivel en particular, algunas/os siendo docentes de nivel medio que decidieron presentarse a concurso en la universidad y siguen haciendo carrera en los dos niveles. Hay también quienes, teniendo su principal actividad en organismos de investigación, decidieron tomar un cargo simple en la universidad, a la vez que hay otras/os que viniendo de dicha actividad, prefirieron quedarse tiempo completo en la universidad por la estabilidad que esta presenta en relación a la carrera de investigación.

Estas diversas experiencias que han transitado las/os docentes entrevistadas/os al ingresar a la UNS, al igual que sus trayectorias académicas dan cuenta que los recorridos por la universidad son diversos, heterogéneos y configuran vivencias docentes singulares. Lo que es interesante destacar para dicho estudio son aquellos sentidos que las/os docentes construyen de su cotidianeidad en la universidad, es decir, aquellos sucesos que son re-significados por cada docente (Larrosa, 2003; Salguero Myers, 2016).

\section{Trabajar en la universidad}

Otro de los ejes que guió las entrevistas fue la experiencia y los sentidos en torno al trabajo cotidiano en la universidad, los desafíos del nivel, las particularidades, las diversas funciones a cumplir dentro de la misma, entre otras cuestiones que según las/os docentes entrevistadas/os hacen a la universidad un ámbito de trabajo único, sumado a la heterogeneidad interdepartamental que genera múltiples culturas organizacionales.

La diferenciación con los demás niveles educativos, e incluso dentro del nivel superior, con los institutos superiores, fue una de las cuestiones mencionada por algunas/os de las/os entrevistadas/os. En esta diferenciación hicieron alusión por ejemplo a la legitimidad que tiene por sí misma la universidad, como expresa esta docente "yo reconozco como valor de la docencia de este nivel, a diferencia de los otros, que tenemos la posibilidad y estamos legitimados para construir conocimiento, seguramente los otros niveles educativos también pero no están legitimados" (Docente Dpto. de Humanidades, 16 años de antigüedad).

Así también valoran de la universidad la posibilidad de vincular docencia e investigación, "algo que agradezco a este sistema es esta posibilidad de mezclar docencia con investigación, esta posibilidad que te permite el sistema público de viajar y perfeccionarte en otras universidades, en otros países, eso es muy 
REVISTA DE LA ESCUELA DE CIENCIAS DE LA EdUCACIÓN, AÑO 17, NRO. 16, VOL. 2, JULIO A DICIEMBRE DE 2021. PÁGINAS 171-180. ISSN 2362-3349 (EN LÍNEA).CONDICIONES, REGULACIONES Y EXPERIENCIAS DE TRABAJO DOCENTE: EL CASO DE LA UNIVERSIDAD NACIONAL DEL SUR. JULIETA RODERA. VERÓNICA SOLEDAD WALKER.

gratificante siempre" (Docente Dpto. de Agronomía, 13 años de antigüedad). Al igual que destacan la posibilidad de dedicarse a la extensión y a la gestión, funciones que son parte del trabajo en la universidad y que para muchas/os es una gran fortaleza, como expresa dicha docente "para mí el trabajo docente no es solo en el aula, para mí el trabajo docente en la universidad es docencia, investigación, extensión, es gestión, no las pienso como funciones escindidas" (Docente Dpto. de Humanidades, 4 años de antigüedad).

En vinculación con este aspecto, según los datos proporcionados por las encuestas, para el $56,8 \%$ de las/os docentes la investigación es la función más valorada en el ámbito universitario, por sobre la docencia, la gestión y la extensión. A pesar de esto, el 66,4\% de las/os docentes le dedica más tiempo a la docencia.

Otra cuestión a subrayar del trabajo en la universidad, que también se mencionó en varias oportunidades es el hecho de tener libertad en las cátedras y en las funciones a desempeñar, como la posibilidad de ser creativas/os y no tener límite alguno. Muchas/os destacan del trabajo en la universidad la autonomía, la oportunidad de crear sus propios caminos dentro de lo académico y poder desenvolverse -mediante investigación o extensión- en el campo en el que se formaron.

La posibilidad de vinculación con la sociedad es otro punto que distingue a la universidad de cualquier otro ámbito educativo y que para muchas/os de las/os entrevistadas/os es la función principal que debe cumplir dicha institución. La importancia de generar un puente entre los conocimientos construidos en esta y la realidad en la que se vive. Para muchas/os "la universidad tiene la obligación de brindarle a la comunidad algo, tiene el deber, no lo concibo desde otro lugar que no sea sí" (Docente Dpto. de Ciencias de la Salud, 11 años de antigüedad)

Teniendo en cuenta que el trabajo constituye un factor de inscripción social (Castel, 2015) y un soporte identitario que opera como fuente de placer y sufrimiento (Dejours y Gernet, 2012; Baudelot y Gollac, 2011), las entrevistas indagaron sobre lo que significa para las/os docentes trabajar en la universidad.

Es así que, muchas/os docentes afirman que es un privilegio trabajar en la universidad, poder dedicarse a la docencia en una institución pública y en el nivel universitario, es totalmente distinto a cualquier otra experiencia en otro nivel educativo. Un Ayudante de Humanidades ( 5 años de antigüedad) afirma "soy docente universitario" y en referencia a la universidad expresa "la lógica es distinta, el trato con los estudiantes es distintos, nadie te fuerza ni te obliga a estar ahí, vos estás porque lo sentís, cuando no lo sentís te levantas y te vas", es decir, aparece nuevamente como positivo la cuestión organizacional, la libertad y la autonomía en distintos ámbitos, además de ser considerado un espacio distinguido y socialmente legitimado.

Estas mismas consideraciones fueron mencionadas en las encuestas realizadas, donde por medio de una pregunta abierta, se consulta ¿cómo definiría o describiría la experiencia de ser docente universitario? En líneas generales, las respuestas fueron positivas: "Satisfactoria", "Muy buena", "Apasionante y enriquecedora", "Placentera", "Gratificante", "Desafiante". Sin embargo, también se manifestaron cuestiones como la mala remuneración, las diversas sobreexigencias del campo, la desvalorización de la actividad docente por sobre la investigación, las dificultades para ascender/progresar en la carrera docente, y en este contexto los inconvenientes propios de la virtualización en la coyuntura actual, sumado a la falta de formación y asesoramiento en el tema.

\section{Virtualización 'forzada' del TDU}

Como último eje a analizar y siendo un tema transversal a todas las entrevistas y encuestas realizadas, se encuentra la virtualización de la educación superior, la educación a distancia en contexto de pandemia o enseñanza remota en contexto de emergencia. Una realidad que repercutió en todos los aspectos educativos y en todos los actores, desde las condiciones laborales, la salud, las dimensiones de tiempo y espacio, las estrategias de enseñanza, el vínculo entre docentes y estudiantes y al interior de los equipos docentes, entre otras consecuencias que contrajo la virtualización forzada del trabajo docente universitario.

En el caso de la UNS se establecieron una serie de resoluciones y documentos emitidos por la Comisión Académica de Educación a Distancia (CAED) donde se ofrecen recursos varios tanto para docentes como para estudiantes. Sin embargo, las dificultades existieron y generaron un gran desafío para todos los actores educativos, en este caso me centraré en la perspectiva de las/os docentes.

Como se mencionó, las condiciones de trabajo fue uno de los grandes cambios que contrajo la virtualidad, ya sea la falta de conectividad y dispositivos electrónicos indicados, el espacio adecuado para trabajar, el tiempo necesario para dedicarle a esta modalidad, la relación entre tareas del hogar y el trabajo docente, entre otras. En este sentido, las/os docentes entrevistadas/os comentan que si bien hay ciertos aspectos de las condiciones de trabajo que ya se encontraban vulnerados antes del confinamiento, lo que ha generado la virtualidad es acrecentarlo, se volvió un trabajo a demanda, pasó a estar "la uni en casa" donde hay que estar disponible las 24 hs., donde no hay corte, ni límite alguno. Esto también se advirtió en las encuestas, para el $79,6 \%$ de las/os docentes la modalidad virtual le insume más tiempo para el desarrollo de la docencia, el $64 \%$ de las/os docentes encuestadas/os consideran que la relación entre actividades de descanso/ocio y trabajo docente es la más difícil de sobrellevar en este contexto, seguida por la relación entre tareas de cuidado en el hogar y trabajo docente, con el $41 \%$. Siendo la disponibilidad de dispositivos electrónicos y la conectividad las condiciones materiales que menos complicaciones produjeron. 
REVISTA De LA Escuela de CiENCIAS DE LA EduCACIÓN, AÑO 17, NRO. 16, VOL. 2, JULIO A DiCIEMBRE DE 2021. PÁGINAS 171-180. ISSN 2362-3349 (EN LÍNEA). CONDICIONES, REGULACIONES Y EXPERIENCIAS DE TRABAJO DOCENTE: EL CASO DE LA UNIVERSIDAD NACIONAL DEL SUR. JULIETA RODERA. VERÓNICA SOLEDAD WALKER

Este cambio impensado de modalidad y por consiguiente de las condiciones de trabajo repercutió arduamente en la salud de las/os docentes, tal como señalan las encuestas se han intensificado malestares como fatiga visual $(61,1 \%)$, dolores articulares y musculares $(54,3 \%)$ y estrés $(46,3 \%)$. Esto generó que muchas/os docentes tuvieran que hacerse de elementos para sobrellevar el cuatrimestre, como expresa esta docente "tuve que salir a comprar una silla porque al tercer día estaba totalmente contracturada, tuve que salir a comprarme una pelota para que mi espalda lo resistiera, tuve que... digo hay muchas cosas que tuve que hacer" (Docente Dpto. de Economía, 34 años de antigüedad). Tanto las condiciones laborales como los problemas de salud en las/os docentes de educación básica, son objeto de estudio desde hace años (Martínez, 2013; García de Fanelli, 2008, Walker, 2016). Sin embargo, los estudios sobre salud y riesgos de trabajo de la docencia universitaria ocupan un lugar relegado.

Otro de los aspectos mayormente mencionado en torno a la virtualización es el vínculo con las/os estudiantes y con las/os colegas, siendo a su vez lo que más añoran de la modalidad presencial.

En lo que refiere al vínculo con las/os estudiantes, desde la perspectiva de las/os docentes, hay una falta de interacción muy grande, a ciertos estudiantes no las/os han podido conocer, en muchos casos no han podido hacer un seguimiento y verles los rostros porque las cámaras están apagadas y tampoco hay participación, es decir, para muchas/os con la virtualización se perdió la posibilidad de diálogo e intercambio. A diferencia de las clases presenciales, no pueden ver sus gestos y no logran un contacto fluido, como expresa este Ayudante de Humanidades (5 años de antigüedad) "leer los rostros para un docente es clave, voy bien, no voy bien, en la cámara uno no lo puede hacer", continua "no sabes cuándo cortar, en la clase vos sabés cuándo alguien quiere empezar a hablar, cuando alguien se levanta, los tiempos son distintos". Sin dudas es un factor muy relevante del proceso educativo que la virtualización ha complejizado. Según las encuestas, para el $52,7 \%$ de las/os docentes, la adecuación de la/s propuesta/s de la/s asignatura/s a la modalidad virtual presentó grandes dificultades con respecto a la dimensión vincular con las/os estudiantes.

Respecto a la dimensión colectiva del trabajo docente, se ha hecho mucho hincapié en la importancia del trabajo en conjunto, más en este contexto, en la posibilidad de repensar las metodologías y las estrategias de enseñanza al modo virtual y de compartir las vivencias. Se valora mucho el intercambio con el equipo docente y tener el espacio para construir conocimiento en conjunto.

Por último, cabe mencionar otro factor que si bien genera heterogeneidad en todo contexto, se ha diversificado aún más en estas circunstancias, y es el vínculo con el conocimiento a enseñar. El tipo de asignatura impartida incide en el ejercicio del trabajo docente (Walker, 2016). Hay docentes que, dependiendo de la disciplina, trabajan con prácticas en terreno/campo, otras/os en laboratorios y otras/os en materias más teóricas. Esta realidad se vio atravesada por la modalidad virtual, ya que como manifiesta este docente, "el trabajo que teníamos que hacer nos cambió totalmente digamos, no es nada que ver... andar caminando el terreno a juntarnos una vez por semana por el zoom" (Docente Dpto. Ciencias de la Salud, 2 años de antigüedad). Lo mismo sucede con docentes que trabajan en los primeros años de las carreras o quienes tienen un gran número de estudiantes a cargo.

\section{Reflexiones finales}

El análisis presentado permitió reconocer la heterogeneidad del trabajo docente universitario, atravesado por múltiples dimensiones como las condiciones laborales, las regulaciones, las cuestiones organizacionales del nivel, las lógicas diferenciadas entre departamentos, los desafíos según categoría y dedicación y el momento de la trayectoria académica en el que se encuentra cada docente. Esta multidimensionalidad da lugar a la configuración de trayectorias que no son lineales.

El estudio se focalizó en las experiencias y sentidos que las/os propios docentes construyen de su trabajo en la universidad, por lo que las entrevistas y encuestas realizadas a docentes de la UNS tuvieron un papel central, permitiendo ahondar en este aspecto.

El contexto en el que se llevó adelante el trabajo -aislamiento social y preventivo obligatorio (ASPO) y distanciamiento social preventivo y obligatorio (DISPO) por la pandemia de COVID-19-, implicó que se pusiera énfasis en la virtualización del trabajo docente, que sin dudas fue el mayor impacto en estos últimos tiempos, no sólo por la falta de experiencia y capacitación en educación a distancia sino también porque como se observa en las entrevistas y encuestas- la modalidad virtual ha visibilizado y profundizado condiciones de trabajo que venían siendo vulneradas.

Esto nos lleva a seguir repensando el trabajo docente universitario como una práctica social, compleja e históricamente situada, que a su vez se encuentra atravesada por un entramado de condiciones, regulaciones y tensiones propias del campo. Es en este espacio donde las/os docentes configuran sus experiencias, en una red de relaciones con otras/os, con diferentes saberes, con instituciones particulares y con la sociedad en general (Walker, 2017).

Considerar a la universidad como espacio laboral y por lo tanto, a las/os docentes como trabajadoras/es, como actores diferencialmente posicionados en el campo (Bourdieu, 1988), implica visibilizar las rutinas y regularidades del trabajo en la universidad con sus ritmos desiguales, fracturas y escisiones. Es decir, hacer visible el trabajo realmente existente, lo no remunerado, las desiguales condiciones de trabajo, la informalidad, flexibilización y precarización que caracteriza al mundo laboral en general y donde la universidad como ámbito de trabajo no queda al margen (Martinez, 2013). 
REVISTA De LA Escuela de CiENCIAS DE LA EduCACIÓN, AÑO 17, NRO. 16, VOL. 2, JULIO A DiCIEMBRE DE 2021. PÁGINAS 171-180. ISSN 2362-3349 (EN LÍNEA).CONDICIONES, REGULACIONES Y EXPERIENCIAS DE TRABAJO DOCENTE: EL CASO DE LA UNIVERSIDAD NACIONAL DEL SUR. JULIETA RODERA. VERÓNICA SOLEDAD WALKER.

\section{Referencias bibliográficas}

Barroso, J. O. (2005). Estado, a educação e a regulação das políticas públicas. Educação \& Sociedade, 26(92), 725-751. https://www.scielo.br/pdf/es/v26n92/v26n92a02.pdf

Baudelot, C. y Gollac, M. (2011). ¿Trabajar para ser feliz? La felicidad y el trabajo en Francia. Buenos Aires: Miño y Dávila; CEIL; Trabajo y Sociedad.

Becher, T. (1989) Academic tribes and territories. Intellectual enquiry and the cultures of disciplines. The Society for Research into Higher Education \& The Open University Press.

Bourdieu, P. (1988) El interés del sociólogo. En: Bourdieu, P. (Ed.), Cosas dichas (108-114). Buenos Aires: Gedlsa

Boyer, E.; Altbach, P. y Whitelaw, M.J. (1994). The Academic Profession. An International Perspective. A Special report. The Carnegie Foundation for the Advancement of Teaching.

Bruner, J. (1985). Universidad y sociedad en América Latina: un esquema de interpretación. Venezuela:UNESCOCRESALC

Bruner, J. y Flisfisch, A. (1989), Los intelectuales y las instituciones de la cultura. México: Universidad Autónoma Metropolitana-Azcapotzalco.

Castel, R. (2015). Las trampas de la exclusión. Trabajo y utilidad social. Buenos Aires: Topia Editorial, Colección fichas para el siglo XXI.

Chiroleu, A. (2002). La profesión académica en Argentina. Boletín P ROEALC. Síntesis Especial América Latina. Mayonesa. Accesible enhttp://www2.uerj.br/proealc

Clark, B. (1983). El sistema de educación superior: una visión comparativa de la organización académica. Ciudad de México: Nueva Imagen; Universidad Futura. UAM.

Dejours, C. y Gernet, I. (2012). Psicopatología del trabajo. Buenos Aires: Miño y Dávila; CEIL; Ministerio de Trabajo de la provincia de Buenos Aires; Trabajo y Sociedad.

Fernández Lamarra, N. y Perez Centeno, C. (2011). Situación actual de la profesión académica universitaria en Argentina. En: N. Mainero (Comp.) Ensayos e investigaciones sobre la problemática universitaria. Argentina: Nueva Editorial Universitaria. UNSL.

Follari, R. y Soms, E. (1980, mayo). Crítica al Modelo Teórico de la Departamentalización [ponencia]. Alternativas Universitarias, UAM-Azcapotzalco, México, http://publicaciones.anuies.mx/pdfs/revista/Revista37 S1A3ES.pdf

García de Fanelli, A. M. (2008). Profesión académica en la Argentina: Carrera e incentivos a los docentes en las Universidades Nacionales. Argentina: CEDES.

García de Fanelli, A. M. y Moguillansky, M. (2014). La docencia universitaria en Argentina. Obstáculos en la carrera académica. Archivos Analíticos de Políticas Educativas, 22(47), http://repositorio.cedes.org/bitstream/123456789/3204/1/10570.pdf

Guzmán Gómez, C. y Saucedo Ramos, C. (2015). Experiencias, vivencias y sentidos en torno a la escuela y a los estudios. Abordajes desde las perspectivas de alumnos y estudiantes. Revista Mexicana de Investigación Educativa, 20(67), 1019-1054. http://www.scielo.org.mx/pdf/rmie/v20n67/v20n67a2.pdf

Krotsch, P. (2001). Educación superior y reformas comparadas. Buenos Aires: Universidad Nacional de Quilmes.

Larrosa, J. (2003) La experiencia y sus lenguajes. Algunas notas sobre la experiencia y sus lenguajes [conferencia]. Seminario Internacional La Formación Docente entre el siglo XIX y XXI. España: Universidad de Barcelona. http://www.bnm.me.gov.ar/giga1/documentos/EL001417.pdf

Marquina, M. (2013). ¿Hay una profesión académica argentina? Avances y reflexiones sobre un objeto en construcción. Revista Pensamiento Universitario, (15), 35-58.

Marquina, M. y Fernández Lamarra, N. (2008, 10-12 de diciembre). La profesión académica en Argentina: Entre la pertenencia institucional y disciplinar [artículo]. V Jornadas de Sociología de la UNLP, Facultad de Humanidades y Ciencias de la Educación, La Plata, Argentina. http://www.memoria.fahce.unlp.edu.ar/trab eventos/ev.6219/ev.6219.pdf

Martínez Funes, D. (2013). El trabajo en la Universidad. Pequeña introducción a un texto para dar batalla... Espacios en Blanco. Revista en Educación, (23),.45-72. https://www.redalyc.org/pdf/3845/384539805004.pdf

Pérez Centeno, C. (2017). El estudio de la profesión académica universitaria en argentina. Estado de situación y $\begin{array}{lllll}\text { perspectivas. } \quad \text { Integración } & \text { Conocimiento, } & 2(7), & 25-255 .\end{array}$ https://revistas.unc.edu.ar/index.php/integracionyconocimiento/article/view/18691/19116

Perkin, H. (1984). The academic profession in the United Kingdom. En: Clark, B. (Ed.) The Academic Profession: National, Disciplinary and institutional Settings,13-59. California: University of California Press.

Rikap, C. (2016). Heterogeneidades y condiciones de trabajo de los docentes universitarios en la Universidad de Buenos Aires. Un estudio comparado de las Facultades de Farmacia y Bioquímica, y Ciencias Económicas. Trabajo y $\begin{array}{lll}\text { Sociedad, } & (27), & 109-137 .\end{array}$ https://www.unse.edu.ar/trabajoysociedad/27\%20RIKAP\%20CECILla\%20\%20Heterogeneidades\%20entre\%2 0los\%20docentes\%20UBA.pdf

Salguero Myers, K. A. (2016). El concepto de experiencia para pensar la educación secundaria urbana. Córdoba: Universidad Nacional de Villa María.

Stake, R. E. (1999). Investigación con estudio de casos. México: Morata.

Tello, A., et. al. (2004). Las Condiciones del Trabajador Docente en la U.N.S.L. Un análisis a partir de la Carrera Docente. Trabalho e Educação, 13(2),153-164.

Toribio, D. E. (1999) La Evaluación de la Estructura Académica. Buenos Aires: CONEAU.

Walker, V. (2017). El trabajo docente universitario como práctica relacional: sujetos, saberes e instituciones. Revista $\begin{array}{lllll}\text { Educación, Lenguaje } \quad \text { Sociedad, } & 14(14), & 1-35 .\end{array}$ https://cerac.unlpam.edu.ar/index.php/els/article/view/2219/2216

Walker, V. (2019, 4-6 de diciembre). Cambios en el trabajo docente universitario: tendencias globales, instituciones y sujetos [ponencia]. II Encuentro Internacional de Educación. Educación Pública: democracia, derechos humanos y justicia social, UNCPBA, Tandil, Argentina 
REVISTA DE LA Escuela de CienCIAS DE LA EdUCACIÓN, AÑO 17, NRO. 16, VOL. 2, JULIO A DICIEMBRE DE 2021. PÁGINAS 171-180. ISSN 2362-3349 (EN LÍNEA). CONDICIONES, REGULACIONES Y EXPERIENCIAS DE TRABAJO DOCENTE: EL CASO DE LA UNIVERSIDAD NACIONAL DEL SUR. JULIETA RODERA. VERÓNICA SOLEDAD WALKER.

Walker, V. S, Menghini, R. A. y Alarcón, S. M. (2016, 30 de noviembre-1 de diciembre). Notas para problematizar el trabajo docente universitario. El caso de la Universidad Nacional del Sur [ponencia]. IV Seminario Nacional de la Red Estrado Argentina. La regulación del trabajo y la formación docente en el siglo XXI, FFyL-UBA, Buenos Aires, Argentina.

Walker, V. S. (2016). El trabajo docente en la universidad: condiciones, dimensiones y tensiones. Perfiles Educativos, 38(153), 105-119. http://www.scielo.org.mx/pdf/peredu/v38n153/0185-2698-peredu-38-153-00105.pdf

\section{Documentos:}

Secretaría de Políticas Universitarias (2015). Anuarios Estadísticos

Universidad Nacional del Sur (2018-2019). Anuarios Estadísticos

Universidad Nacional del Sur (1996). Res. AU-4/96. Estatuto de la Universidad Nacional del Sur. Texto ordenado. 